LAWRENCE LIVERMORE NAT IO N A L LABORATORY

\title{
Preconditioned MoM Solutions for Complex Planar Arrays
}

B. J. Fasenfest, F. Capolino, D.R. Wilton, D.R. Jackson, N. Champagne

March 30, 2004

2004 IEEE International Symposium on Antennas and Propagation and USNC/URSI National Radio Science Meeting Monterey, CA, United States June 20, 2004 through June 26, 2004 
This document was prepared as an account of work sponsored by an agency of the United States Government. Neither the United States Government nor the University of California nor any of their employees, makes any warranty, express or implied, or assumes any legal liability or responsibility for the accuracy, completeness, or usefulness of any information, apparatus, product, or process disclosed, or represents that its use would not infringe privately owned rights. Reference herein to any specific commercial product, process, or service by trade name, trademark, manufacturer, or otherwise, does not necessarily constitute or imply its endorsement, recommendation, or favoring by the United States Government or the University of California. The views and opinions of authors expressed herein do not necessarily state or reflect those of the United States Government or the University of California, and shall not be used for advertising or product endorsement purposes. 


\title{
Preconditioned MoM Solutions for Complex Planar Arrays
}

\author{
B. J. Fasenfest ${ }^{1}$, F. Capolino ${ }^{2}$, D.R. Wilton ${ }^{1}$, D.R. Jackson ${ }^{1}$, and N. Champagne ${ }^{3}$ \\ 1) ECE Dept., University of Houston, Houston, TX 77204, USA. \\ 2) Dept. of Information Engineering., University of Siena, 53100 Siena, Italy. \\ 3) Lawrence Livermore National Lab., Livermore, CA, USA. ${ }^{\dagger}$
}

\section{Introduction}

The numerical analysis of large arrays is a complex problem. There are several techniques currently under development in this area. One such technique is the FAIM (Faster Adaptive Integral Method). This method uses a modification of the standard AIM approach [1] which takes into account the reusability properties of matrices that arise from identical array elements.

If the array consists of planar conducting bodies, the array elements are meshed using standard subdomain basis functions, such as the RWG basis. These bases are then projected onto a regular grid of interpolating polynomials. This grid can then be used in a 2D or 3D FFT to accelerate the matrix-vector product used in an iterative solver [2]. The method has been proven to greatly reduce solve time by speeding the matrix-vector product computation. The FAIM approach also reduces fill time and memory requirements, since only the near element interactions need to be calculated exactly.

The present work extends FAIM by modifying it to allow for layered material Green's Functions and dielectrics. In addition, a preconditioner is implemented to greatly reduce the number of iterations required for a solution. The general scheme of the FAIM method is reported in [2]; this contribution is limited to presenting new results.

\section{Layered Material}

To apply FAIM, the moment matrix for the original discretized EFIE, $\left[Z_{m n}^{\mathbf{p p}^{\prime}}\right]\left[\mathrm{I}_{n}^{\mathbf{p}^{\prime}}\right]=\left[\left(V_{m}^{\mathbf{p}}\right)^{\mathbf{i n c}^{\mathbf{n c}}}\right]$, is written as [2]

$$
\left[\Delta Z_{m n}^{\mathbf{p p ^ { \prime }}}\right]\left[\mathrm{I}_{n}^{\mathbf{p}^{\prime}}\right]+\left[\tilde{Z}_{m n}^{\mathbf{p p ^ { \prime }}}\right]\left[\mathrm{I}_{n}^{\mathbf{p}^{\prime}}\right]=\left[\left(V_{m}^{\mathbf{p}}\right)^{\mathbf{i n c}}\right]
$$

where the block Toeplitz difference matrix $\Delta Z_{m n}^{\mathbf{p p}{ }^{\prime}}=Z_{m n}^{\mathbf{p p}{ }^{\prime}}-\tilde{Z}_{m n}^{\mathbf{p p}{ }^{\prime}}$ is taken as zero for elements whose indices satisfy $\left|\mathbf{p}-\mathbf{p}^{\prime}\right| \geq \mathbf{c}$ and is hence sparse. Concerning notation, as shown in Fig.1, the array is decomposed into blocks of elements with each element denoted by the two-component multiindex $\mathbf{p}$, with a prime added to distinguish source from observation element locations. Within each block representing an element, the current is expressed in terms of usual basis functions $\Lambda_{n}^{\mathbf{p}^{\prime}}$. The $m$-indexed test functions are denoted by $\Lambda_{m}^{\mathbf{p}}$ (see [2] for more details.) To evaluate the matrix/vector product, we note that $\left[\Delta Z_{m n}^{\mathbf{p p}^{\prime}}\right]\left[\mathrm{I}_{n}^{\mathbf{p}^{\mathbf{p}^{\prime}}}\right]$ is quickly computed since $\Delta Z_{m n}^{\mathbf{p p}^{\prime}}$ is sparse, whereas $\left[\tilde{Z}_{m n}^{\mathbf{p p}^{\prime}}\right]\left[\mathrm{I}_{n}^{\mathbf{p}^{\prime}}\right]$ is of convolutional form and can be evaluated using a 2D FFT as follows:

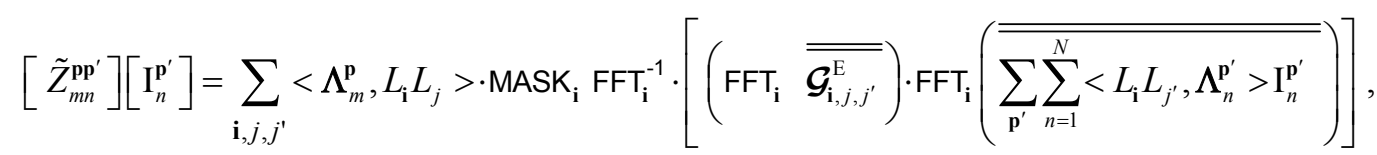

${ }^{\dagger}$ This work was performed under the auspices of the U.S. Department of Energy by University of California, Lawrence Livermore National Laboratory under Contract W-7405-Eng-48. 
where $\mathbf{p}$ and $\mathbf{p}$ ' represent the two-index displacements between source and observation cells, respectively; $m$ and $n$ index unknowns within the $\mathbf{p}$ and $\mathbf{p}$ ' cells, respectively; and $\mathbf{i}, j$ and $\mathbf{i}^{\prime}, j^{\prime}$ denote periodic grid points for the Green's function evaluations (Fig.1). $\left\langle\Lambda_{m}^{\mathbf{p}}, L_{\mathbf{i}} L_{j}\right\rangle$ is the projection of the $m$ th basis function in the pth array cell onto the Lagrange polynomial interpolating the $\mathbf{i}, j$ th point. $\mathcal{G}_{\mathbf{i}, j, j^{\prime}}^{\mathrm{E}}$ represents the sampled Green's electric field dyad, though in actuality the field is expressed in mixed potential form. In free space, the dyad can be expressed in terms of a single scalar potential. Since vector basis functions are used, $<\Lambda_{m}^{\mathrm{p}}, L_{\mathbf{i}} L_{j}>$ is a vector.

For layered material, the far interactions require the computation and storage of the five non-zero components of the magnetic vector potential Green's dyad and two scalar potentials for all possible interactions between interpolating points in the $z$ dimension, and all unique discrete separations in the transverse dimension. This increases memory requirements when layered material is present, but does not increase the number of FFT's that need to be performed per iteration.

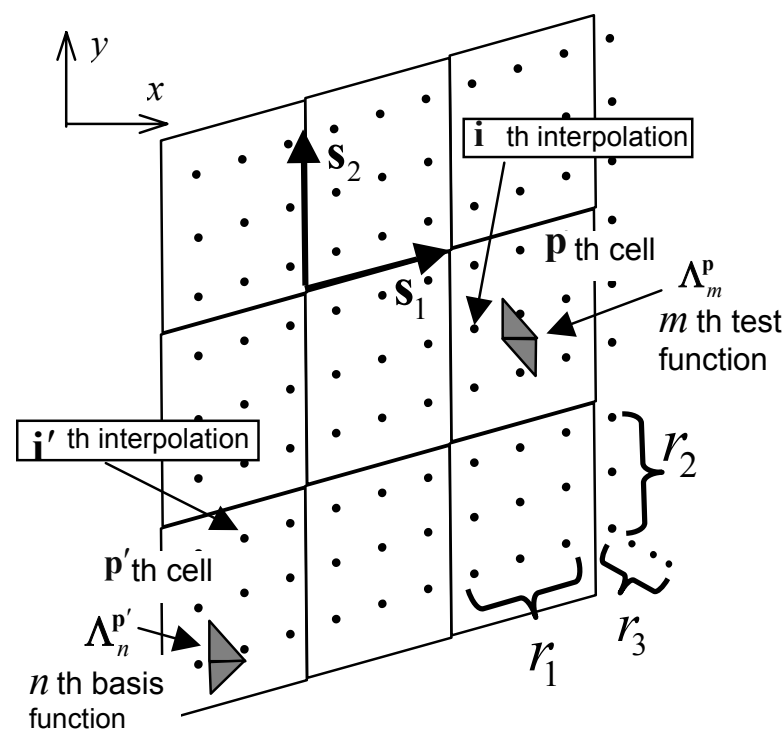

Fig. 1. Array cell index definitions and arbitrary skew lattice vectors. The periodic grid on which the Green's function is evaluated and sampled is shown superimposed on the array cells. Within an array cell, the Green's function is evaluated at $r_{1} \times r_{2} \times r_{3}$ points.
The Green's function samples themselves are only transformed once, before any iterations are performed. During each iteration, the currents are projected onto the interpolating grid as usual. However, once the projections are transformed into the spectral domain, more than one multiplication per vector component is completed, applying each of the Green's function's dyadic terms. The inverse transform is then computed, and the iteration completed. Because multiplications in the spectral domain are $\mathrm{O}(N)$ while the transforms themselves are $\mathrm{O}(N \log N)$, the extra Green's function terms do not greatly slow the iteration.

\section{Dielectrics}

For dielectric array elements, the PMCHWT surface formulation is used. The original EFIE equation is then replaced by a matrix system of the following form:

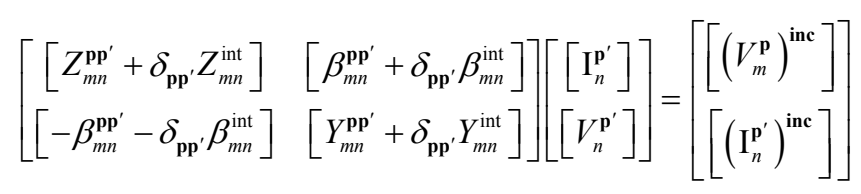

The matrix $Z_{m n}^{\mathrm{pp}^{\prime}}$ is the EFIE operator and $Y_{m n}^{\mathbf{p p}^{\prime}}$ is its dual, representing the magnetic field due to magnetic current sources; $\beta_{m n}^{\text {pp' }}$ is the corresponding MFIE operator. The corresponding matrices $Z_{m n}^{\text {int }}, Y_{m n}^{\text {int }}$, and $\beta_{m n}^{\text {int }}$ that appear only on diagonal blocks represent the coupling from the interior of 
each dielectric element; they affect only the $\mathbf{p}=\mathbf{p}^{\prime}$ blocks of the near-field terms of the FAIM approach comprising blocks such that $\left|\mathbf{p}-\mathbf{p}^{\prime}\right|<\mathbf{c}$.

\section{Block Diagonal Preconditioner}

When using an iterative solver such as BiCGStab on a very large matrix system, the solution may converge very slowly if conditioning is poor. For this reason, a preconditioner is needed to improve the solution time.

A block diagonal preconditioner for an array is a logical and simple choice. This preconditioner consists of the diagonal (self-array cell) terms of the impedance matrix only. The inverse of this matrix is another block-diagonal matrix containing the inverse of the self-array cell blocks $\left[Z_{m n}^{\mathrm{pp}^{\prime}}\right]$, with $\mathbf{p}=\mathbf{p}^{\prime}$. Physically, this preconditioner solves the original problem as if there were no interaction between array cells. For array designs with little mutual coupling this is a very good assumption and the iteration count can be reduced by orders of magnitude. For arrays with strong coupling some deterioration in performance is to be expected.

Because the self block is already being filled exactly for use in the correction calculation, this preconditioner does not require additional fill time. The inversion of a self block is also negligible since the number of unknowns involved is small compared to the overall array size. After the matrix-vector product is computed during each iteration, the resulting vector is multiplied by the preconditioner, adding an $\mathrm{O}(N)$ computation to the total time for the matrix vector product.

\section{Results}

Three test arrays were simulated with different structures and the results of the FAIM method both with and without preconditioning were compared to an "exact" MoM solution of these arrays. The "exact" solution does not use interpolation or fast multiplication, but does utilize the Toeplitz nature of the matrices to speed fill time and storage.

The first two arrays consisted of $20 \times 20$ elements, had a lattice spacing of a half wavelength in both directions, and were simulated at $300 \mathrm{MHz}$. The first test case was a dipole fed by a delta gap source at its center. The dipole contained 23 unknowns and was $39[\mathrm{~cm}]$ long and $1[\mathrm{~cm}]$ wide. The second test case involved the same dipole on a grounded dielectric slab. The height of the dielectric slab was $19[\mathrm{~cm}]$ and its relative permittivity was 2.55 . Both these cases used fourth order interpolation of the Green's Function.

The final case was an array of $25 \times 25$ square conducting patches in free space illuminated by a plane wave at $6 \mathrm{GHz}$ incident from a direction perpendicular to the array plane. The patches were $11.4[\mathrm{~mm}]$ on a side with a spacing of $\mathrm{S}_{1}=\mathrm{S}_{2}=3.8[\mathrm{~mm}]$ between patches. Each patch was meshed using triangles, creating 65 unknowns per patch. This FAIM method used fifth order interpolating polynomials in both planar directions.

Table 1 shows the run times for the exact and FAIM solutions of the three arrays, as well as the error in the FAIM solution compared to that of the full moment matrix. It can be clearly seen that the FAIM method offers a dramatic savings in both fill and solve times while maintaining a high level of accuracy. It can also be seen that use of the preconditioner drastically reduces the number of iterations needed for a solution, further reducing solution time. 
Table 1: Matrix fill and solve times for FAIM and exact method for several structures.

\begin{tabular}{|c|c|c|c|c|}
\hline & $\frac{\text { Fill Time }}{\text { [s] }}$ & $\frac{\text { Solve }}{\text { Time [s] }}$ & $\frac{\text { Number }}{\text { Iterations }}$ & $\frac{\text { Average \% }}{\text { Error }}$ \\
\hline Dipole in Free Space & 608.0 & 1591.2 & 309 & \\
\hline FAIM & 4.2 & 232.0 & 263 & 0.20 \\
\hline FAIM w/ preconditioner & 4.2 & 7.2 & 7 & 0.19 \\
\hline $\begin{array}{l}\text { Dipole on Grounded } \\
\text { Substrate }\end{array}$ & 4698.6 & 4297.1 & 833 & \\
\hline FAIM & 47.1 & 1132.7 & 911 & 0.15 \\
\hline FAIM w/ preconditioner & 47.6 & 23.2 & 17 & 0.15 \\
\hline Square Patch in Free Space & 4391.8 & 53612.1 & 463 & \\
\hline FAIM & 27.7 & 1100.5 & 340 & 0.96 \\
\hline FAIM w/ preconditioner & 27.0 & 32.0 & 9 & 0.97 \\
\hline
\end{tabular}

As indicated in the table, the FAIM method also drastically reduces the memory storage requirements. For example, for the $25 \times 25$ square patch array, each patch was discretized using $N=65$ basis function, requiring a storage of $N \times N=4225$ complex numbers for each $\mathbf{p}, \mathbf{p}^{\prime}$ block $\left[Z_{m n}^{\mathbf{p p}^{\prime}}\right]$ of the impedance matrix. Instead, using FAIM with a fifth order interpolation scheme, we need store only 25 Green's function samples per cell. For layered media, this number must be multiplied by the number of unique dyadic and scalar potential terms used in the mixed potential formulation. FAIM's storage advantage is further amplified by the fact that if there are $M$ array cells in a square array, there are $M^{2}$ matrix blocks in the complete matrix, while there are only about $4 M$ blocks of sampled Green's function points. For the $25 \times 25$ array, this means that the system matrix for a standard solution must contain about $1.6 \times 10^{9}$ complex entries, while there are only $62.5 \times 10^{3}$ entries in the sampled Green's function array.

\section{Conclusion}

The FAIM method for solving large arrays has been extended to arbitrary array elements, including dielectrics and layered material. The addition of both features fit neatly into the FAIM method, requiring only additional Green's function terms and projections for magnetic currents. A block diagonal preconditioner has been tested and found to greatly improve solve time by reducing the number of iterations required by the BiCG solver.

\section{Bibliography}

[1] E. Bleszynski, M. Bleszynski, and T. Jaroszewicz, "AIM: Adaptive integral method for solving large scale electromagnetic Scattering and Radiation Problems," Radio Science, v. 31, pp. 1225$1251,1996$.

[2] B. Fasenfest, F. Capolino, D.R. Wilton, and D. R. Jackson, N. Champagne, "General MoM Solutions for Large Arrays", ICEAA (Int. Conf. Electromagnetics and Advanced Appl.), Torino, Italy, 8-12 September, 2003. Also accepted for IEEE Antennas and Propagat. Wireless Letters. 